\title{
3 Research Square \\ Sister Mary Joseph Nodules: A Case Report About a Rare Location of Skin Metastasis
}

\section{Brice Leyrat}

Centre Jean Perrin

Maureen Bernadach

Centre Jean Perrin

\section{Angeline Ginzac}

Centre Jean Perrin

Sejdi Lusho ( $\nabla$ sejdi.lusho@clermont.unicancer.fr)

Centre Jean PERRIN https://orcid.org/0000-0002-2359-6308

\section{Xavier Durando}

Centre Jean Perrin

\section{Case Report}

Keywords: Umbilical skin metastases, Sister Mary Joseph nodules, Pancreatic adenocarcinoma, Chemotherapy, Oncology, Case report

Posted Date: January 11th, 2021

DOI: https://doi.org/10.21203/rs.3.rs-141436/v1

License: (c) (i) This work is licensed under a Creative Commons Attribution 4.0 International License. Read Full License 


\section{Abstract}

\section{Background}

Umbilical skin metastases (or Sister Mary Joseph nodules) are rare. Their presence typically indicates the late manifestation of deep-seated abdominopelvic malignancy. They occur mainly in gynecological cancers, and gastro-intestinal cancers in men. The most common histology is adenocarcinoma ( 75\% of cases), but it can also rarely be squamous cell or undifferentiated carcinoma. These metastases can be present at diagnosis or appear at disease recurrence, and are associated with a very poor prognosis with an average survival of 11 months.

\section{Case presentation}

We report the clinical case of a 58-year-old man with metastatic pancreatic adenocarcinoma and umbilical cutaneous metastasis after receiving first-line chemotherapy. The diagnosis was established upon liver biopsy in July 2019, after the patient presented with complaint of transfixiant abdominal pain. The first-line treatment consisted of six cycles of modified FOLFIRINOX chemotherapy. However, in November 2019, computed tomography (CT) scan showed disease progression. Second-line treatment with gemcitabine (brand name: Gemzar) led to a $16 \%$ decrease of target lesions. During the fourth cycle, three periumbilical indurated nodules appeared. After six cycles, skin infiltration had increased and the patient reported his abdominal pain had intensified. Reassessment by CT scan showed an increase in both hepatic and peritoneal disease progression. Third-line treatment with FOLFIRI, started on April 15, 2020, was not able to control the disease, leading to greater induration and subcutaneous infiltration, which were responsible for the increased pain and ultimate death.

\section{Conclusion}

Umbilical skin metastases are rare and they are associated with advanced metastatic disease and a very poor prognosis. Cases reporting Sister Mary Joseph nodules are needed to better understand the conditions and mechanisms of their appearance and dissemination.

\section{Introduction}

Umbilical skin metastases, also known as Sister Mary Joseph nodules, are rare. They are described in 1$3 \%$ of patients with neoplasia of abdominopelvic origin (gynecological or digestive) ${ }^{[1,2]}$. Umbilical metastases are most common in gastric cancer (30\%), colorectal cancer $(25 \%)$ and pancreatic cancer $(18 \%)$ in men, and in ovarian cancer (34\%), endometrial cancer $(12 \%)$, and colorectal cancer $(12 \%)$ in women. There is a predominance of females who present with these nodules (male to female ratio of 0.69 ); however, the majority of male cases have digestive etiologies ${ }^{[1,2]}$. In $14-33 \%$ of cases, the discovery of umbilical metastasis leads to the diagnosis of an unknown neoplasia ${ }^{[3]}$. In contrast, among those patients with known neoplasia, the umbilical nodules are an early sign of recurrence in about $40 \%$ and in about $59 \%$ of cases, the umbilical metastases occur after discovery of the primary tumor ${ }^{[3]}$. 
They can be present at diagnosis or appear at disease recurrence, and are associated with a very poor prognosis, having an average survival of 11 months ${ }^{[4]}$. Their presence typically indicates the late manifestation of deep-seated abdominopelvic malignancy.

Here, we report an original case of a patient with metastatic pancreatic adenocarcinoma at diagnosis, who developed periumbilical nodules during the course of his disease.

\section{Case Presentation}

\section{Diagnosis}

A 58-year-old man presented with transfixiant abdominal pain on July 17, 2019. A corporeo-caudal lesion of the pancreas, containing the celiac trunk and associated with secondary hepatic lesions, was discovered in July 2019.

The patient had no symptoms, signs or findings prior to the abdominal pain that prompted presentation. Upon hospital admission the patient underwent a physical examination by an oncologist.

Regarding the personal history of past illnesses, the patient had undergone surgery and radiation therapy for a testicular seminoma in 2007.

Laboratory testing was performed and showed that plasma angiotensin-converting enzyme level was elevated $(17.3 \mathrm{ng} / \mathrm{mL}$; normal: $<5.2 \mathrm{ng} / \mathrm{mL})$ and the carbohydrate antigen $19-9$ level was exceedingly elevated $(7329 \mathrm{UI} / \mathrm{mL}$; normal: $<27 \mathrm{UI} / \mathrm{mL}$ ). Liver biopsy confirmed the diagnosis of pancreatic adenocarcinoma and ruled out the possible recurrence of testicular seminoma. No deleterious mutations were identified in the tumor tissue for the breast cancer $1 / 2(B R C A 1 / 2)$ genes.

Computed tomography (CT) scan showed secondary hepatic lesions and peritoneal carcinomatosis. The final diagnosis was corporeo-caudal lesion of the pancreas, containing the celiac trunk and associated with secondary hepatic lesions.

\section{Treatment}

Figure 1 illustrates the treatment timeline.

\section{First-line treatment}

The patient received modified FOLFIRINOX (5-fluorouracile $1200 \mathrm{mg} / \mathrm{m}^{2}$ continuously for $46 \mathrm{~h}$, levofolinate $200 \mathrm{mg} / \mathrm{m}^{2}$, oxaliplatin $85 \mathrm{mg} / \mathrm{m}^{2}$, irinotecan $150 \mathrm{mg} / \mathrm{m}^{2}$ ) as first-line chemotherapy ${ }^{[5]}$. After six cycles, in November 2019, the CT scan revealed an increase in pre-existing liver lesions as well as the appearance of new liver lesions and lesions of peritoneal carcinomatosis, indicating disease progression according to Response Evaluation Criteria in Solid Tumors (RECIST) 1.1. An incidental pulmonary embolism was also discovered. 


\section{Second-line treatment}

Second-line treatment $\left(1250 \mathrm{mg} / \mathrm{m}^{2}\right)$ was administered on days 1 and 8 , and repeated on day 21 , adapted to the patient's general condition ${ }^{[6]}$. After three cycles of treatment, the disease was deemed stable, according to the RECIST 1.1 criteria (i.e. a 16\% decrease in target hepatic and pancreatic lesions). However, during the fourth cycle of gemcitabine (Gemzar ${ }^{\circledR}$; Eli Lilly), three periumbilical indurated nodules appeared, two of which were erythematous (Fig. 2A). During the fifth cycle of gemcitabine, these nodules remained visible and slightly increased in size (Fig. 2B). After six cycles, skin infiltration and abdominal pain associated with peritoneal carcinomatosis increased. The umbilical orifice showed unfolding of the umbilicus (Fig. 2C). Reassessment by CT scan also showed disease progression (according to RECIST 1.1), both hepatic and peritoneal.

\section{Third-line treatment}

Third-line treatment with FOLFIRI (5-fluorouracil bolus $400 \mathrm{mg} / \mathrm{m}^{2}$ and then $1200 \mathrm{mg} / \mathrm{m}^{2}$ administered continuously for $46 \mathrm{~h}$, irinotecan $180 \mathrm{mg} / \mathrm{m}^{2}$ ) was started on April 15, $2020{ }^{[7]}$. Under FOLFIRI, there was clinical progression of the periumbilical lesions (Fig. 3); in particular, greater induration and subcutaneous infiltration occurred, which were responsible for increased pain.

\section{Outcome and follow-up}

Unfortunately, the patient died from his illness on July 11, 2020, after receiving all three lines of treatment.

\section{Discussion}

The most common histology of umbilical skin metastases is adenocarcinoma (accounting for $~ 75 \%$ of cases), but it can also (rarely) be squamous cell carcinoma or undifferentiated cancer ${ }^{[2]}$. Because of its vascularization, connections to embryological remnants, and proximity to the peritoneum, the umbilicus is predisposed to being a site of metastasis. As such, several hypotheses have been put forth to explain the underlying pathophysiology ${ }^{[2]}$. These mainly concern the umbilical region receiving rich arterial vascularization via the inferior epigastric artery and the iliac circumflex artery arising from the external iliac artery and via the superior epigastric artery arising from the internal mammary artery.

Under normal conditions, venous drainage occurs through the internal mammary vein to the axillary vein, and through the lower epigastric vein to the femoral vein. The umbilical region is also drained by a lymphatic system that follows the venous system. It comprises a deep network (involving para-aortic, internal mammary and external iliac nodes) and a superficial network (involving axillary and inguinal nodes $)^{[8]}$. Lymphatic diffusion appears to be the most likely mechanism of dissemination, exerting a major impact on prognosis and surgical management ${ }^{[2]}$. 
Tumor cells undergo both anterograde and retrograde diffusion, making the umbilicus a point of intersection of the lymphatic network ${ }^{[2,9]}$. In addition, the umbilicus is separated from the peritoneum only by a single structure (the fascia transversalis) which may be strengthened by focal thickening of the

fascia umbilicalis ${ }^{[2,8]}$. This close connection between the umbilicus and anterior peritoneal surface may allow contiguous diffusion. The umbilicus is a scar that appears after birth when the umbilical cord falls off but retains connection to the embryonic remains, including Meckel's diverticulum (a remnant of the vitelline duct), the falciform ligament (from the umbilical veins, connecting the vena cava), the urachus (itself a remnant of the allantoid duct, connecting the umbilicus to the roof of the bladder), and the medial umbilical ligaments (on the sides of the urachus, arising from the umbilical arteries) ${ }^{[2]}$. Despite the many anatomical connections of the embryonic remnants, they do not seem to play an important role in the dissemination of tumor cells. The presence of an umbilical nodule is usually associated with advanced metastatic disease and, therefore, with a very poor prognosis (average survival of only $11 \mathrm{mo}$ ) $[2,10]$.

\section{Conclusion}

In this case study, we followed a patient, whose umbilical metastases appeared after first-line treatment, showing aggressive disease and a poor prognosis. Their appearance is strongly linked to the progression of peritoneal carcinomatosis, suggesting a mixed mechanism of dissemination, most likely by lymphatic diffusion and promiscuity.

\section{Abbreviations}

CT Computed tomography

RECIST Response Evaluation Criteria in Solid Tumors

\section{Declarations}

Ethics approval and consent to participate

The patient passed away on July 11, 2020 and written consent could not be obtained. However, the patient had not previously opposed his data to be used for publication in a case report.

Consent for publication

The patient had not previously opposed his data to be used for publication in a case report. According to French laws and regulations, patient data can be used for research and published after the patient's death, if the patient did not previously oppose to such use.

Availability of data and materials 
Not applicable

Competing interests

The authors declare that the research was conducted in the absence of any commercial or financial relationships that could be construed as a potential conflict of interest.

Funding

Not applicable

Authors' contributions

$B L, M B$, and $X D$ were the patient's oncologists and were involved in patient's case management. $B L$ and MB designed and wrote the case report. MB, SL, and XD were responsible for revising the manuscript for important intellectual content. SL and AG were responsible for the manuscript layout and submission. All authors read and approved the final manuscript and were involved in the review and editing of the manuscript.

Acknowledgements

Not applicable

\section{References}

1. Touraud JP, Lentz N, Dutronc Y, Mercier E, Sagot P, Lambert D. [Umbilical cutaneous metastasis (or Sister Mary Joseph's nodule) disclosing an ovarian adenocarcinoma]. Gynecol Obstet Fertil 2000;28(10):719-21.

2. Dubreuil A, Dompmartin A, Barjot P, Louvet S, Leroy D. Umbilical metastasis or Sister Mary Joseph's nodule. Int J Dermatol 1998;37(1):7-13.

3. Galvañ VG. Sister Mary Joseph's nodule. Ann Intern Med 1998;128(5):410.

4. De Angeli M, Carosi M, Vizza E, Corrado G. Sister Mary Joseph's nodule in endometrial cancer: A case report and review of the literature. J Cancer Res Ther 2019;15(6):1408-10.

5. Conroy T, Paillot B, François E, Bugat R, Jacob J-H, Stein U, et al. Irinotecan plus oxaliplatin and leucovorin-modulated fluorouracil in advanced pancreatic cancer-a Groupe Tumeurs Digestives of the Federation Nationale des Centres de Lutte Contre le Cancer study. J Clin Oncol Off J Am Soc Clin Oncol 2005;23(6):1228-36.

6. Burris HA, Moore MJ, Andersen J, Green MR, Rothenberg ML, Modiano MR, et al. Improvements in survival and clinical benefit with gemcitabine as first-line therapy for patients with advanced pancreas cancer: a randomized trial. J Clin Oncol Off J Am Soc Clin Oncol 1997;15(6):2403-13.

7. Neuzillet $C$, Hentic $O$, Rousseau B, Rebours V, Bengrine-Lefèvre L, Bonnetain F, et al. FOLFIRI regimen in metastatic pancreatic adenocarcinoma resistant to gemcitabine and platinum-salts. World $\mathrm{J}$ 
Gastroenterol 2012;18(33):4533-41.

8. Ching ASC, Lai CW. Sonography of umbilical metastasis (Sister Mary Joseph's nodule): from embryology to imaging. Abdom Imaging 2002;27(6):746-9.

9. Glover RP, Waugh JM. The retrograde lymphatic spread of carcinoma of the rectosigmoid region; its influence on surgical procedures. Surg Gynecol Obstet 1946;82:434-48.

10. Gabriele R, Conte M, Egidi F, Borghese M. Umbilical metastases: current viewpoint. World J Surg Oncol 2005;3(1):13.

\section{Figures}

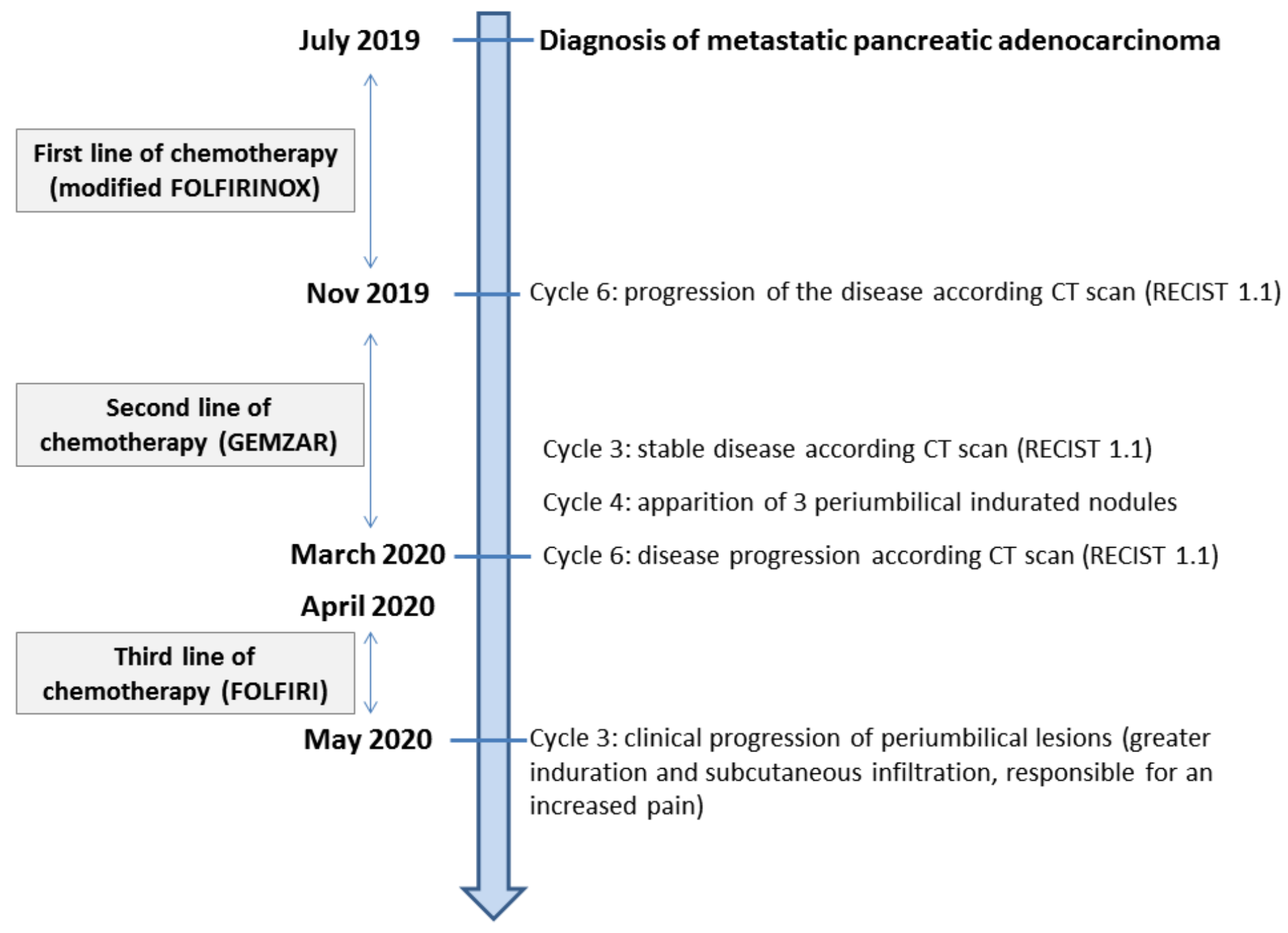

Figure 1

Treatment timeline. 


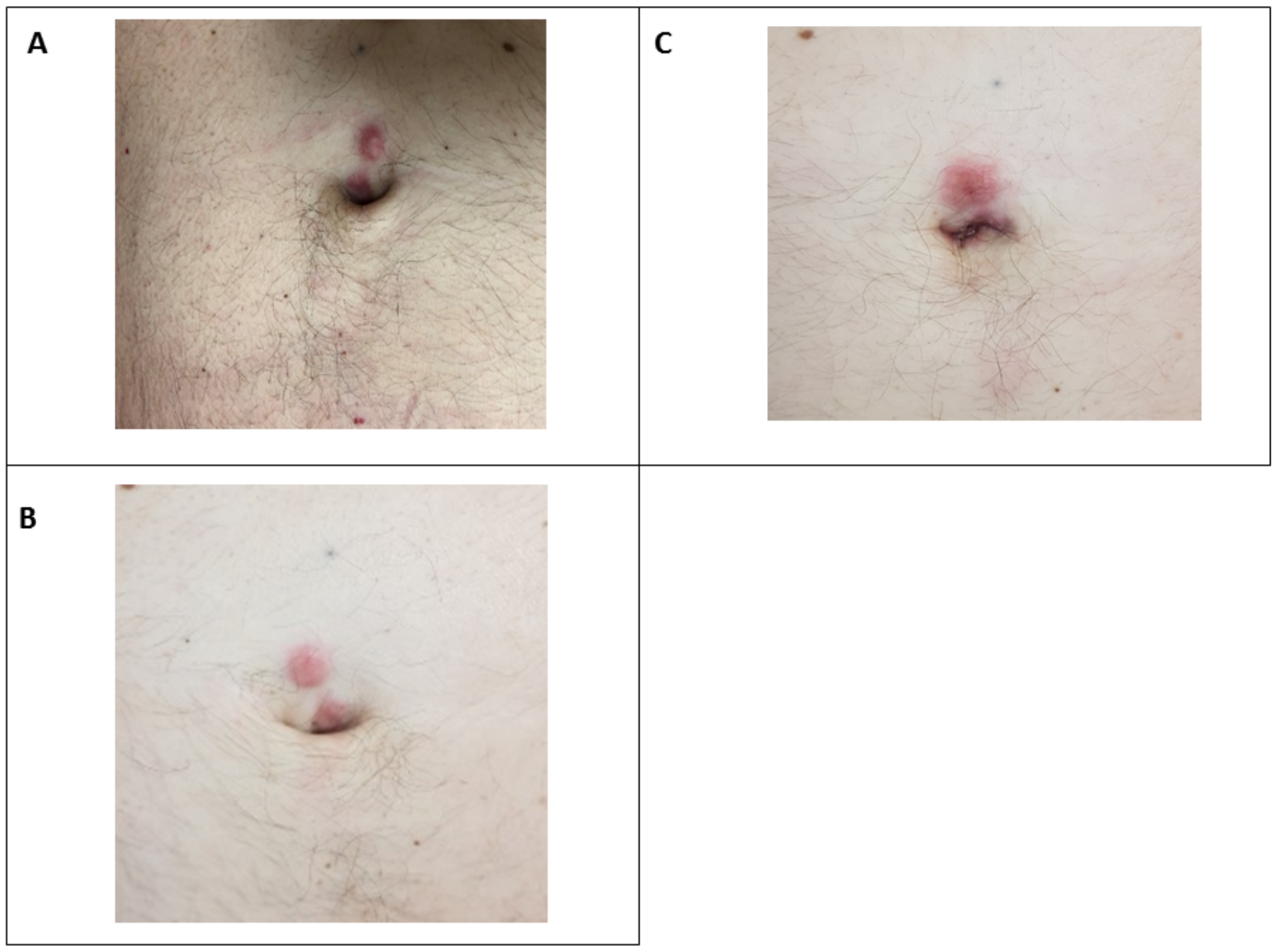

Figure 2

Evolution of the metastatic periumbilical nodules of pancreatic adenocarcinoma throughout the Gemzar ${ }^{\circledR}$ treatment. A: Fourth cycle of Gemzar ${ }^{\circledR}$, started on February 12, 2020; B: Fifth cycle of Gemzar®, started on March 4, 2020; C: Sixth cycle of Gemzar®, started on March 25, 2020. 


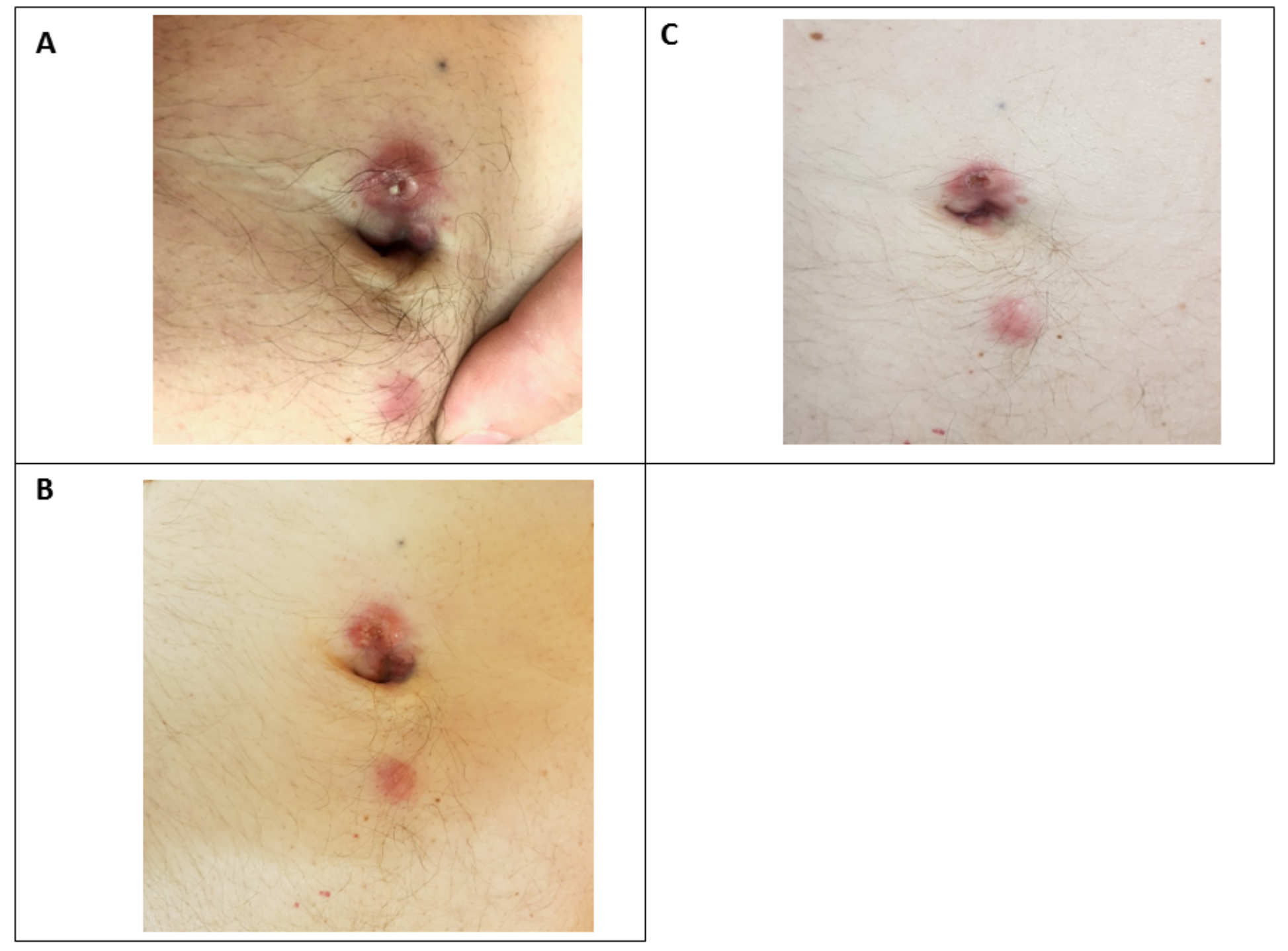

\section{Figure 3}

Evolution of the metastatic periumbilical nodules of pancreatic adenocarcinoma throughout the FOLFIRI treatment. A: First cycle of FOLFIRI, started on April 15, 2020; B: Second cycle of FOLFIRI, started on April 28, 2020; C: Third cycle of FOLFIRI, started on May 12, 2020. 